Pacific Journal of Mathematic 


\section{ACTIONS OF FINITE GROUPS ON SELF-INJECTIVE RINGS ${ }^{1}$}

\section{Handelman and G. Renault}

Let $G$ be a finite group of automorphisms of a ring $R$ (with 1), and suppose the order of $G$ is not a zero diviser in $R$. We denote by $R^{G}$ the subring of $R$ consisting of elements fixed pointwise by each member of $G$. We consider, for a class of rings, the questions whether $R$ viewed as a right (or left) $R^{G}$-module is finitely generated, and how the type classification of $R$ and $R^{G}$ relate when $R$ is self-injective regular.

Even in the case of a commutative noetherian ring $R, R$ need not be finitely generated over $R^{\alpha}$, as shown by Chuang and Lee [1]. However, if $R$ is a finite product of simple rings, more generally if $R$ is biregular, then the finite generation does hold. The proof utilizes the skew group ring $R_{S} G$ and an elementary result from Morita theory; as a consequence, we obtain a short, easy proof of the theorem of Farkas and Snider [3; Theorem 1], for $R$ semisimple artinian.

For self-injective rings $R$, the finite generation need not hold: nevertheless the techniques involved in the biregular case can be used to show that the type classifications are preserved. In otherwords, $R$ and $R^{G}$ are simultaneously of types $I_{f}, I_{\infty}, I I_{f}, I I_{\infty}$, or $I I I$. This completes work of the second author [14].

If $R$ is self-injective regular, then $R$ is injective as an $R^{G_{-}}$ module, and we show that $R$ is projective if and only if it is finitely generated. This is done by showing that any nonsingular injective module over a self-injective regular ring that is also projective, must be finitely generated, or else the ring has an artinian ring direct summand.

This work was almost entirely done during both authors' stay at the Summer Research Institute of the Canadian Mathematical Congress, at the University of Waterloo in the summer of 1978. The second author would particularly like to thank V. Dlab and J. Lawrence for their hospitality.

I. Biregular rings. A convenient tool for dealing with group actions, is the skew group ring. Let $G$ be a group, with an action as automorphisms on $R$. Form the free left $R$-module with basis $G, R_{s} G$, equipped with multiplication extended $R$-linearly from

${ }^{1}$ In an earlier version, this paper was written in French, under the title, "Actions de Groupes". 


$$
r g=g r^{g} \text { for } r \text { in } R, g \text { in } G .
$$

See for example [7].

THEOREM 1. Let $R$ be a finite product of simple rings, and suppose $G$ is a finite group acting as automorphisms of $R$, with the order of $G$ invertible in $R$. Then $R_{s} G$, the skew group ring, is also a finite product of simple rings.

REMARK. The proof below is due to the referee of the earlier version of this paper, viz, footnote 1 . Other proofs were independently given by D. Passman, J. Fisher, and the authors.

Proof. Let $A$ be a finite product of simple rings. The bimodule (two-sided ideal) structure of $A$ is reflected in its semisimplicity as a left $A \bigotimes_{z} A^{o p}$-module, with the action $\left(\sum a_{i} \otimes b_{j}\right)(a)=\sum a_{i} a b_{j}$.

Setting $A=R_{1} \times \cdots \times R_{n}=R$, with the $R_{i}$ all simple, $R_{S} G$ as an $R \otimes R^{o p}$-module, is a direct sum of the simple $R \otimes R^{o p}$-modules, $R_{i} g, g$ varying over $G$, so $R_{S} G$ is semisimple as an $R \otimes R^{o p}$-module.

It suffices to show that every two-sided ideal $I$ of $R_{S} G$ is a retract of $R_{S} G$, as a bimodule, equivalently as an $R_{S} G \otimes\left(R_{S} G\right)^{o p}$ bimodule.

Since $R_{S} G$ is $R \otimes R^{o p}$-semisimple, and $I$ is a submodule, there is an $R \otimes R^{o p}$-linear (i.e., an $R$-bimodule homomorphism) retraction $v: R_{S} G \rightarrow I$. Define the two-sided analogue of the usual averaging process,

$$
V: R_{S} G \longrightarrow I
$$

by setting

$$
V(x)=\frac{1}{|G|^{2}} \sum_{a, h \in G} g v\left(g^{-1} x h^{-1}\right) h .
$$

One routinely checks that $V$ is an $R_{S} G$-bimodule homomorphism, fixing $I$ pointwise.

For $A$ any ring, $Z(A)$ will denote its center.

COROLlary 2. Let $R$ be a biregular self-injective ring, and $G$ a finite group acting as automorphisms, with the order of $G$ invertible in $R$. Then $R_{S} G$ is also biregular and self-injective.

REMARKS. "Self-injective" means right-injective unless otherwise specified. The corresponding result with self-injectivity deleted from the hypothesis and conclusion is false. 
Proof. It is well-known that $R_{S} G$ is self-injective (and regular), so by [13, Proposition 1.6], it suffices to show that every prime ideal of $R_{S} G$ is maximal.

Let $P$ be a prime ideal of $R_{S} G$, and observe that $(Z(P))^{G} /(P \cap$ $\left.(Z(R))^{G}\right)$ embeds naturally in the center of $R_{S} G / P$, so must be a field. Since $Z(R)$ is a commutative biregular ring and $Z(R) /(P \cap$ $Z(R))$ is finitely generated over that field, $\left(P \cap(Z(R))^{d}\right) Z(R)$ is a finite intersection of maximal ideals of $Z(R)$. Since this ideal is contained in $Z(R) \cap P$, the latter is also such an intersection. From the biregularity of $R$, we deduce that $(Z(R) \cap P) R$ is a finite intersection of prime, hence maximal, ideals of $R$. As this ideal is contained in $R \cap P$, the latter is also a finite intersection.

Clearly, $R \cap P$ is a $G$-invariant ideal of $R$, and we may thus form the skew group ring $(R /(R \cap P))_{s} G$; there is a natural mapping of rings from this onto $\left(R_{s} G\right) / P$. By Theorem 1 , the former is a finite product of simple rings, so the latter being prime, must be simple.

The following lemma is a standard result from Morita theory, and is a special case of $[2 ; 1,4.1 .3]$.

LEMma 3. Let $A$ be a ring, and let $P$ be a finitely generated projective $A$-module that is a generator for $\operatorname{Mod}-A$. Set $B=\operatorname{End}_{A} P$. Then $P_{B}$ is a finitely generated projective module.

THEOREM 4. Let $R$ be a ring, and $G$ a finite group of automorphisms of $R$, with the order of $G$ invertible in $R$. If either (1) or (2) below hold,

(1) $R$ is a finite product of simple rings

(2) $R$ is a biregular, self-injective ring then $R$ is a finitely generated projective $R^{G}$-module.

Proof. As in [4], consider the $R_{s} G-R^{G}$ bimodule, $R$. As a left $R_{s} G$-module, $R$ is projective and isomorphic to the principal left ideal $R_{s} G e$, where $e=|G|^{-1} \sum g$. Since $R_{s} G$ is biregular (by the first two results), there exists a central idempotent $F$ such that the ideal $Q=F \cdot R_{s} G$ is the left annihilator of $R_{s} G e$, hence of the left module $R$. So $R$ is an $\left(R_{s} G / Q\right)-R^{G}$ bimodule in a natural way; it is faithful, projective, and finitely generated over $R_{s} G / Q$.

Any finitely generated faithful projective module $P$ over a biregular ring $S$ is a generator: There exists an integer $n$ so that $P \simeq e S^{n}$ (for some $e=e^{2}$ in $M_{n} S$ ); since $M_{n} S$ is biregular, $M_{n} S e M_{n} S$ is generated by a central idempotent $E$, but $P$ will not be faithful if $E$ is not the identity; hence $M_{n} S e M_{n} S=M_{n} S$, so $e S^{n} \simeq P$ is a generator. 
Thus $R$ as a left $R_{s} G / Q$-module is a generator, so if $E=$ $\operatorname{End}_{R_{s} G / Q} R, R_{E}$ is finitely generated projective. However, there is an isomorphism of $E$ with $R^{G}$ so that the action of $E$ on $R$ is translated to the usual action of $P^{G}$ on $R$. Thus $R$ is finitely generated projective as an $R^{G}$-module.

COROLlaRY 5 [9]. If $R$ is a finite product of simple rings, and $G$ is a finite group of automorphisms of $R$ with the order of $G$ invertible in $R$, then $R^{G}$ is also a finite product of simple rings.

Proof. As is implicit in the proof of Theorem $4, R^{G} \simeq e R_{s} G e$ (same $e$ ), and Theorem 1 applies.

The theorem of Farkas and Snider [3; Theorem 1] asserts that $R$ is a finitely generated $R^{G}$-module if $R$ is semisimple artinian (and the usual condition on the order of $G$ ). This is of course a special case of Theorem 4, but is easier to prove as it only requires proving Theorem 1 for the special case, $R$ semisimple artinian (which is just Maschke's theorem).

II. Self-injective regular rings. We may now complete the results of Renault [14] on the relation between the type classifications of $R$ and $R^{G}$, when $R$ is self-injective. Specifically, we show that $R$ is type II (respectively type $\mathrm{II}_{\infty}$ ) if and only if $R^{G}$ is, and since the corresponding result is known for type $I_{f}$ (and type $I_{\infty}$ ), it also holds for type III. For a review of the type classification for self-injective regular rings, see $[5 ; \S 7]^{2}$

Proposition 6. Let $R$ be a regular self-injective ring, dnd $G$ a finite group of automorphisms of $R$, with the order of $G$ invertible in $R$. Then

(1) $R$ is of type II (respectively $\mathrm{II}_{f}$ ) if and only if

(2) $R^{G}$ is of type II (respectively type $\mathrm{II}_{f}$ ).

Proof. According to [14; Corollary 10], (1) implies (2). So it suffices to show (2) implies (1).

Let $e$ be any finite idempotent in $R^{G}$; then $(e R e)^{G}=e R^{G} e$, and we shall show $e$ is finite as an idempotent in $R$.

Let $M$ be a maximal two-sided ideal of $S=e R e$, and set $P=$ $\cap M^{g}$. Now $S / P$ is a finite prduct of simple rings, so by Theorem $4, S / P$ is finitely generated as a module over $(S / P)^{G}$, which equals

${ }^{2}$ A comprehensive treatment occurs in the very recently published von Neumann Regular Rings, by K. R. Goodearl, published by Pitman (1979). 
$S^{(} /\left(P \cap S^{G}\right)$, and being projective as well, $S / P$ embeds in a corner of a matrix ring over $S^{G} /\left(P \cap S^{G}\right)$. Since quotients, matrix rings, and corners of directly finite self-injective rings are also directly finite, $S / P$ is directly finite. Since $S / M$ is one of the simple ring direct summands of $S / P, S / M$ is also thus directly finite.

So for all maximal two-sided ideals $M$ of $S, S / M$ is directly finite; it is easily checked that for self-injective rings, this implies $S$ is directly finite (outline of proof: if not, there is a central idempotent $E$ such that $T=E S$ satisfies, $T \oplus T \simeq T$ as $T$-modules; this property is inherited by all homomorphic images of $T$ ). Thus the idempotent $e$ is finite in $R$.

If $R^{G}$ were of type $\mathrm{II}_{f}$, we may set $e=1$, so that $R=S$ is of finite type, and it is easy to check that $R^{G}$ having no artinian images implies the same for $R$; thus $R$ is of type $\mathrm{II}_{f}$. If $R$ is merely of type II, there exists a faithful finite idempotent $e$ in $R^{a}$, and to show $R$ is of type II, it suffices to show that $e$ is faithful in $R$.

If not, the right (and left) annihilator of $R e R$ is $E R$ for some central idempotent $E$. Since $R e R$ is $G$-invariant, so is $E R$; since $E^{g}$ must also be central, it follows that $E^{g}=E$, so $E$ belongs to $R^{G}$. As $e$ is a faithful idempotent in $R^{G}, E=0$.

We can also show that for self-injective rings $R, R^{G}$ is biregular if and only if $R$ is. We first require a slight extension of [13; Proposition 1.6].

Proposition 7. A self-injective regular ring all of whose primitive images are simple, is biregular.

Proof. Let $M$ be a maximal ideal of the center, $Z(R)$ of $R$. The quotient ring $T=R / M R$ has its two-sided ideals totally ordered [12; Prop. 2.9]. Let $N$ be a two-sided ideal of $R$ properly containing $M R$; since $T$ is regular, there is a maximal right ideal $Q$ of $R$ containing $M R$ but not $N$. Inside $Q$ is a primitive ideal containing $M R$, but not $N$; as $Q$ must be maximal and the ideals of $T$ are totally ordered, this is a contradiction. Hence, no such $N$ exists, so $M R$ is a maximal two-sided ideal. Thus all maximal ideals of $R$ are of the form $M R$, so $R$ is biregular [13; Prop. 1.1].

THEOREM 8. Let $R$ be regular and self-injective. Suppose G $i s$ a finite group of automorphisms of $R$, and the order of $G$ is invertible in $R$. Then

(1) $R$ is biregular

if and only if

(2) $R^{G}$ is biregular. 
Proof. Since $R^{G} \simeq e R_{s} G e$ (as in the proofs of Theorem 4 and Corollary 5), (1) implies (2).

Assume (2) holds. Let $P$ be a primitive ideal; by the preceding result, it suffices to show $P$ is maximal. Form $Q=\cap P^{g}$, and the quotient ring $T=R / Q$. As $Q$ is $G$-invariant, we may form $T_{s} G$, which is also, in a natural may, an image of $R_{s} G$.

Since $R_{s} G$ is self-injective and regular, it satisfies general comparability: [5; Theorem 3.3]. ${ }^{2}$

$\left.{ }^{*}\right)$ For all idempotents $e, f$ there exists a central idempotent $E$ such that the right ideal generated by $e E$ is subisomorphic to that generated by $f E$, and the right ideal generated by $f(1-E)$ is subisomorphic to that generated by $e(1-E)$.

Since subisomorphisms between idempotent-generated principal right ideals are equationally determined in a regular ring, $\left(^{*}\right)$ is inherited by all homomorphic images of $R_{s} G$; in particular $T_{s} G$ satisfies $\left(^{*}\right)$.

Now consider the center of $T_{s} G$ : routine computations (as in [7; 1.6 proof (1), (2)] - observe that since $T_{s} G$ is regular, nonzero divisors are invertible), show that the center is contained in a finitely generated module over the center of $T$. However, $T$ is a finite product of prime regular rings ( $T$ is a subdirect product of finitely many prime rings, but satisfies $\left.\left({ }^{*}\right)\right)$, so $Z(T)$ is a finite product of fields; it easily follows that $Z\left(T_{s} G\right)$ is artinian. As $T_{s} G$ is a regular ring satisfying (*), it follows that $T_{s} G$ is also a finite product of prime rings.

As $T^{G} \simeq e T_{s} G e, T^{G}$ is a finite product of prime rings. Since $T^{G}$ is a homomorphic image of a biregular ring $\left(R^{G}\right), T^{G}$ is thus a finite product of simple rings.

Thus $T^{G}$ is a finite product of simple rings and $T$ is a finite product of prime rings; by $[4 ; 4.3], R / Q=T$ is a finite product of simple rings, whence $R / P$ is simple.

We note that in the course of the above proof, we have shown:

COROLlaRY 9. If $R$ is a finite product of prime regular selfinjective rings and $G$ is a finite group of automorphisms with the order of $G$ invertible in $R$, then both $R^{G}, R_{s} G$ are finite products of prime regular self-injective rings.

The main idea involved in the proofs of Corollary 2 and Theorem 8 is that prime ideals are maximal in $R_{s} G$ if they are so in $R$. Now that the results of $[11 ; 1.4]$ are available, shorter proofs can be given.

The following result, interesting in itself, is useful for determining the connection between the projectivity and the finite gener- 
ation of $R$ as an $R^{i}$-module, which we shall now be exploring.

THEOREM 10. Let $R$ be a right self-injective regular ring, and I a nonsingular injective right module. At least one of the following hold:

(a) I is finitely generated;

(b) There exists a strictly descending infinite sequence of central idempotents $F_{1}>F_{2}>\cdots$ such that

\section{$\oplus F_{i} R$ is subisomorphic to $I$;}

(c) There exists a nonzero central idempotent $E$ such that $\aleph_{0}$ copies of the module ER is subisomorphic to $I$.

REMARK. We adopt the notation $n M$ or $n(M)$ to indicate a direct sum of $n$ copies of $M$, when $M$ is a module and $n$ a positive integer or $\aleph_{0}$.

Proof. We repeatedly use general comparability, that is, for $J$ a nonsingular injective over $R$, there exists a central idempotent $E$ with $J E \lesssim E R$ and $(1-E) R \lesssim J(1-E)$ (all as right modules) [5; Theorem 3.3] .

Assume neither (a) nor (c) hold. There is a central idempotent $E_{1}$ of $R$ with $I E_{1} \lesssim E_{1} R$ and $\left(1-E_{1}\right) R \lesssim I\left(1-E_{1}\right)$. As (a) fails, $E_{1}$ does not equal 1 , and from the negation of (c), there exists a positive integer $n_{1}$ such that $n_{1}\left[\left(1-E_{1}\right) R\right] \lesssim I\left(1-E_{1}\right)$, but $\left(n_{1}+\right.$ 1) $\left[\left(1-E_{1}\right) R\right] \not L I\left(1-E_{1}\right) R$. Since all the modules dealt with are injective, all these subisomorphisms split, so there exists an injective submodule $K_{1}$ with $I\left(1-E_{1}\right) \simeq K_{1} \oplus n_{1}\left[\left(1-E_{1}\right) R\right]$.

Set $F_{1}=1-E_{1}$, and view $K_{1}$ as a module over $F_{1} R$. There exists a central idempotent $E_{2}<F_{1}$ such that $K_{1} E_{2} \lesssim E_{2} R$ and $\left(F_{1}-\right.$ $\left.E_{2}\right) R \lesssim K_{1}\left(F-E_{2}\right)$. Now $F_{1} R$ is not subisomorphic to $K_{1}$, so $E_{2}$ is not zero. On the other hand, there exists, by the negation of (c) a positive integer $n_{2}$ with $n_{2}\left(F_{1}-E_{2}\right) R \lesssim K_{1}\left(F_{1}-E_{2}\right)$ but no larger number of copies can be embedded in $K_{1}\left(F_{1}-E_{2}\right)$. Write $K_{1}\left(F_{1}-\right.$ $\left.E_{2}\right) \simeq n_{2}\left(F-E_{2}\right) R \oplus K_{2}$ for some $K_{2}$. Set $F_{2}=F_{1}-E_{2}$; this process can obviously be continued inductively, and we obtain $\oplus n_{i} F_{i} R \lesssim I$, and the $F_{i}$ are strictly descending. This verifies (b).

THEOREM 11. Let $R$ be a right self-injective regular ring, and $I$ a projective injective right $R$-module. Then there is a decomposition

$$
I=J \oplus K
$$

where $J$ is finitely generated, $K=$ Socle $(K)$, and there is a central 
idempotent $E$ with $K E=K$, and $E R$ is artinian.

(For this proof only, we distinguish between the internal direct sum $(\oplus)$, and the external direct sum $(\Perp)$.)

Proof. We repeatedly employ the following idea: If $\left\{e_{i} R\right\}$ is an infinite collection of nonzero principal right ideals, and $\Perp e_{i} R \lesssim R$, then $\Perp e_{i} R$ cannot be injective. For, the image of $\Perp e_{i} R$ is an internal direct sum $\oplus f_{i} R \cong R$; being injective, it must be a direct summand of $R$, and hence would be principal; but this is impossible since the generator would have to appear in a finite direct summand.

Since $I$ is projective, it (and all of its submodules) is nonsingular. Let $K_{1}$ be the injective hull of the socle of $I$; there is a direct summand $J_{1}$ so that $J_{1} \oplus K_{1}=I$, and of course Socle $\left(J_{1}\right)=\{0\}$. We proceed to show that $J_{1}$ must be finitely generated.

Being projective (and $P$ being regular), $J_{1}$ is isomorphic to a direct sum of principal right ideals of $R$, say $J \simeq \Perp e_{i} R$, where $e_{i}=e_{i}^{2}$ belong to $R$. Either $10(\mathrm{~b})$ or $10(\mathrm{c})$ holds, and we show either leads to a contradiction, unless the index set is finite.

If 10 (c) holds, we may find inside the index set infinitely many disjoint finite subsets $\left\{S_{j}\right\}$ such that $E R \lesssim \Perp_{i \in S_{j}} e_{i} R$ for some idempotent $E$, for all $j$. By passing to a direct summand of $J_{1}$, and multiplying by the central idempotent $E$, we may assume $E=1$, and $\cup S_{j}$ is the entire index set, so that for all $j$,

$$
R \lesssim \underset{i \in S_{j}}{\mathbb{\Perp}} e_{i} R \text {. }
$$

Since $J_{1}$ is a faithful $E R$-module and Socle $\left(J_{1}\right)=\{0\}, E R$ has zero socle, and in particular, is not artinian. So we may find an infinite orthogonal set of idempotents $\left\{f_{j}\right\}$ in $R$, in bijection with the set of $S_{j}$ 's. For each $j$, there is an $i$ in $S_{j}$ and a nonzero idempotent $g_{j}$ in $e_{i} R$ with $g_{j} R \lesssim f_{j} R$. There thus exists an idempotent $h_{j}$ in $f_{j} R$ with $g_{j} R \simeq h_{j} R$. As $\Perp g_{j} R$ is a direct summand of $J_{1}$, a contradiction arises unless $J_{1}$ is finitely generated.

Now suppose $10(\mathrm{~b})$ holds. We may find infinitely many finite disjoint subsets $\left\{S_{j}\right\}$ of the index set, and an infinite sequence of descending central idempotents $\left\{F_{j}\right\}$ such that for all $j, F_{j} R \lesssim$ $\Perp_{i \in S_{j}} e_{i} R$. Then $\left(F_{j}-F_{j+1}\right) R \lesssim \Perp_{i \in S_{j}} e_{i} R$; set $E_{j}=F_{j}-F_{j+1}$, note that the $E_{j}$ are orthogonal, and $\Perp E_{j} R$ is isomorphic to a direct summand of $J_{1}$, so is injective, and again the first paragraph applies to yield a contradiction. Hence $J_{1}$ must be finitely generated.

Write $K_{1}=\Perp_{i \in T} h_{i} R, h_{i}=h_{i}^{2} \in R$. Then for each $i$, Socle $\left(h_{i} R\right)$ is essential in $h_{i} R$. Let $S$ be a set of representatives of isomorphism classes of minimal right ideals of $R$ that appear in Socle $\left(K_{1}\right)$. Suppose there is an infinite subset $T_{0}$ of $T$, an infinite subset $S_{0}$ of $S$, 
and a bijection $f: T_{0} \rightarrow S_{0}$ so that for $t$ in $T_{0}, f(t)=g_{t} R \lesssim h_{t} R$. Then from the minimality of each of the $g_{t} R$ (and their mutual nonisomorphism), $\Perp g_{t} R \cong \bigoplus g_{t} R \cong R$; but $\Perp g_{t} R$ is isomorphic to a direct summand of $K_{1}$ (each $g_{t} R$ is a direct summand of $h_{t} R$ ), and again a contradiction occurs.

In particular, we deduce that for at most finitely many $t$ in $T$ can $h_{t} R$ contain infinitely many elements of $S$. By absorbing these into the finitely generated $J_{1}$, we may assume all of the $h_{t} R$ contain only finitely many members of $S$. Knowing this, we deduce from the previous paragraph that $S$ must be finite, say $S=\left\{g_{1} R, g_{2} R, \cdots\right.$, $g_{n} R$. Then if $E(\quad)$ denotes injective hull, $K_{1}=E\left(\bigoplus^{n} N_{i} g_{i} R\right) \cong \Perp$ $E\left(N_{i} g_{i} R\right), N_{i}$ finite or infinite cardinals. Whichever of the $E\left(N_{i} g_{i} R\right)$ are finitely generated can be absorbed into the finitely generated part, and we are reduced to the situation, $K \simeq \Perp E\left(N_{i} g_{i} R\right)$, where each of the $E\left(N_{i} g_{i} R\right)$ is not finitely generated, and the $g_{i} R$ are mutually nonisomorphic.

Let $F_{i}$ be the central cover of $g_{i} R$. Then $E\left(N_{i} g_{i} R\right)$ is a projective injective module over $T_{i}=F_{i} R$. Since $g_{i} R$ is a faithful irreducible $T_{i}$-module, $T_{i}$ is primitive, and so $10(\mathrm{~b})$ cannot apply to $E\left(N_{i} g_{i} R\right)$; as $E\left(N_{i} g_{i} R\right)$ is not a finitely generated $R$-module, it cannot be finitely generated as a $T_{i}$-module; thus $10(\mathrm{c})$ must apply. If $T_{i}$ contained an infinite orthogonal set of idempotents, we could apply the process applied to $J_{1}$ to reach a contradictoin - thus $T_{i}$ must be artinian, and so $E=\sum E_{i}$ generates an artinian corner of $R$. Since $E R$ is artinian, $K$ is completely reducible as an $E R$; hence as an $R$-module.

Now if $R$ is regular self-injective, and $G$ is a finite group of automorphisms of $R$ with the usual order condition, then $R$ is injective as a right $R^{G}$-module. If $R$ were projective as well, modulo artinian direct summands (which can be dealt with separately), it would have to be finitely generated. On the other hand, if $R$ were finitely generated as an $R^{G}$-module, the argument of the following lemma shows $R$ must also be projective.

Lemma 12. Suppose $A$ is a right self-injective regular ring, and $P$ is a faithful finitely generated projective left $A$-module. Set $B=\operatorname{End}_{A} P$, and suppose $P_{B}$ is finitely generated. Then ${ }_{A} P$ is a projective generator for $A$-Mod.

Proof. It is routine to check that $P_{B}$ is nonsingular, and also that $B$ is itself right self-injective, so being finitely generated, $P_{B}$ must be projective ([5; Theorem I. 16]). There thus exists a split onto map of right $B$-modules, 


$$
n B_{B} \longrightarrow P_{B} \text {. }
$$

Applying the contravariant functor, $\operatorname{Hom}\left(-,{ }_{A} P_{B}\right)$, we obtain a split embedding, $n\left({ }_{A} P\right) \leftarrow{ }_{A}$ End $\left(P_{B}\right)$.

Now the natural map $A \rightarrow$ End $\left(P_{B}\right), a \mapsto \hat{a}, \hat{a}(p)=a p$, has kernel the annihilator of ${ }_{A} P$, and so is an embedding. Thus ${ }_{A} A$ embeds in $n\left({ }_{A} P\right)$. Since ${ }_{A} P,{ }_{A} A$ are projective modules over a regular ring, ${ }_{A} A$ is a direct summand of $n\left({ }_{4} P\right)$, and so ${ }_{A} P$ is a generator.

Lorenz and Passman have given examples of type $I_{f}$ self-injective regular rings with $G$ of order 2 (and $1 / 2$ belongs to $R$ ) such that $R$ is not finitely generated over $R^{G}$. We now present complementary examples, with $R$ prime (and necessarily not simple, by Theorem 4).

Example. $\quad R$ prime regular self-injective, not simple, $G=\{1, g\}$ a group of automorphisms of order 2 , with $R$ neither finitely generated nor projective over $R^{G}$.

Take any prime, nonsimple self-injective regular ring with 2 invertible (examples of type $I_{\infty}$ and III exist in profusion; examples of type $\mathrm{II}_{\infty}$ also exist, but require some subtlety to construct). Let $M$ be the (unique, proper) maximal two-sided ideal of $R$, and pick a nonzero idempotent $e$ in $M$.

Let $g$ be the inner automorphism defined as conjugation by $1-2 e$; so $g^{2}$ is the identity, and $R^{G}=e R e \times(1-e) R(1-e)$. If $R$ were $R^{G}$-finitely generated on the right, multiplication by $e$ yields that $R e$ is finitely generated as a right $e R e$-module. Now $R e$ is a faithful projective left $R$-module, so by Lemma $12,{ }_{R} R e$ would have to be a generator, and of course this implies $R e R=R$; but $R e R$ is contained in $M$, a contradiction.

If $R$ were $R^{G}$-projective, it would have to be finitely generated by Theorem 11 .

The automorphism in the example was inner. Not surprisingly, when $G$ consists of outer automorphisms, and $R$ is prime regular self-injective, $R$ is finitely generated over $R^{G}$. Here, 'outer' means not conjugation by an invertible element (the usual definition, as opposed to the ersatz definitions).

THEOREM 14. Let $R$ be a prime regular self-injective ring, and suppose $G$ is a finite group of outer automorphisms of $R$, with the order of $G$ invertible in $R$. Then $R$ is finitely generated and projective as a right $R^{G}$-module, and $R^{G}$ is a prime regular right self-injective ring. 
Proof. We observe that $R_{s} G$ is self-injective, regular, and computing the center as in $[7 ; 1.6$ proof of (2)] (noting that in a prime ring $r R=R r \neq\{0\}$ implies $r$ is not a zero-divisor, and nonzero divisors in regular rings are invertible), we find the center is a field, $Z(R)^{G}$. Since $R_{s} G$ satisfies central comparability, it must be prime (also a special case of $\left[15 ; 2.6\right.$ (ii)]); as $R^{G}$ is isomorphic to a corner of $R_{s} G, R^{G}$ is also prime (and regular, self-injective).

Now let $M$ be the unique maximal two-sided ideal of $R$. Since $M$ is the only maximal two-sided ideal, it must be $G$-invariant, and thus $M R_{s} G$ is a two-sided ideal of $R_{s} G$. The natural isomorphism, $R_{s} G / M R_{s} G \simeq(R / M)_{s} G$ carries a prime ring (the ideals of $R_{s} G$ are totally ordered, so all images are prime) to a finite product of simple rings (Theorem 1), so both are simple.

The idempotent $e=|G|^{-1} \sum g$ has nonzero image in the simple ring $R_{s} G / M R_{s} G$, and as $M R_{s} G$ must be the unique maximal ideal of $R_{s} G$, it follows that $R_{s} G \mathrm{e} R_{s} G=R_{s} G$. Thus $R_{s} G$ is Morita equivalent to $R^{G}$ via the bimodule $R_{s} G e$, and after translating $R_{s} G e$ to $R$, we can apply Lemma 3, as in the proof of Theorem 4 .

We would like to thank Martin Lorenz and Don Passman for pointing out a blunder in the earlier version of this paper.

\section{REFERENCES}

1. C. L. Chuang and P. H. Lee, Noetherian rings with involution, Chinese J. of Mathematics, 5 (1977).

2. C. Faith, Algebra: Rings, Modules and Categories I, Springer Verlag New York, 1973.

3. D. Farkas and R. Snider, Noetherian fixed rings, Pacific J. Math., 69 (1977), 347-353.

4. J. Fisher and J. Osterburg, Semiprime ideals in rings with finite group actions, J. Algebra, 50 (1978), 488-502.

5. K. R. Goodearl and A. K. Boyle, Dimension Theory for Nonsingular Injective Modules, Memoirs Amer. Math. Soc.' 177 (1976).

6. D. Handelman, Algèbres simples de groupes à gauche, Canad. J. Math., 32 (1980), 165-184.

7. D. Handelman, J. Lawrence and W. Schelter, Skew group rings, Houston J. Math., 4 (1978), 175-190.

8. D. Handelman, Perspectivity and cancellation in regular rngs, J. of Algebra, 48 (1977), $1-16$.

9. V. K. Kharchenko, Galois subgroups of simple rings, Math. Zametki, 17 (1975), 887892.

10. - Generalized identities with automorphisms, Algebra and Logic, 14 (1975), $132-148$.

11. M. Lorenz and D. Passman, Prime ideals in crossed products of finite groups,

12. G. Renault, Anneaux réguliers auto-injectifs à droite, Bul. Soc. Math. France, 101 (1973), 237-254.

13. - Anneaux biréguliers auto-injectifs à droite, J. Algebra, 36 (1975), 77-84.

14. - Actions de groupes et anneaux réguliers injectifs, Proc, Waterloo Ring Theo-

ry Conf. (1978), Springer Lecture Notes in Mathematics, 734 (1979).

15. S. Montgomery and D. Passman, Crossed products over prime rings, Israel J. Math.,

31 (1978), 224-256. 
Received May 8, 1979 and in revised form September 10, 1979. (The first author was supported in part by NSERC (formerly, NRC of Canada).

UNIVERSITY OF OtTAWA

Ottawa, Ontar io

CANADA, KIN 9B4 


\section{PACIFIC JOURNAL OF MATHEMATICS}

\section{EDITORS}

DONALD BABBITT (Managing Editor)

University of Galifornia

Los Angeles, California 90024

Hugo RossI

University of Utah

Salt Lake City, UT 84112

C. C. MoOre AND ANDrew OGG

University of California

Berkeley, CA 94720

\section{J. DugundjI}

Department of Mathematics University of Southern California

Los Angeles, California 90007

R. Finn and J. Milgram

Stanford University

Stanford, California 94305

ASSOCIATE EDITORS

E. F. Beckenbachi

B. H. NeumanN

F. WOLF

K. YOSHIDA

\section{SUPPORTING INSTITUTIONS}

UNIVERSITY OF BRITISH COLUMBIA

CALIFORNIA INSTITUTE OF TECHNOLOGY

UNIVERSITY OF CALIFORNIA

MONTANA STATE UNIVERSITY

UNIVERSITY OF NEVADA, RENO

NEW MEXICO STATE UNIVERSITY

OREGON STATE UNIVERSITY

UNIVERSITY OF OREGON
UNIVERSITY OF SOUTHERN CALIFONIA

STANFORD UNIVERSITY

UNIVERSITY OF HAWAII

UNIVERSITY OF TOKYO

UNIVERSITY OF UTAH

WASHINGTON STATE UNIVERSITY

UNIVERSITY OF WASHINGTON

Printed in Japan by International Academic Printing Co., Ltd., Tokyo, Japan 


\section{Pacific Journal of Mathematics \\ Vol. 89 , No. 1 \\ May, 1980}

David Bressoud, A note on gap-frequency partitions ................ 1

John David Brillhart, A double inversion formula ................. 7

Frank Richard Deutsch, Günther Nürnberger and Ivan Singer, Weak

Chebyshev subspaces and alternation .......................

Edward Richard Fadell, The relationship between Ljusternik-Schnirelman

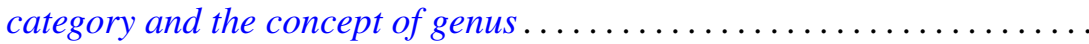

Harriet Jane Fell, On the zeros of convex combinations of polynomials.......

John Albert Fridy, An addendum to: "Tauberian theorems via block

dominated matrices" ..................................

Andrzej Granas, Ronald Bernard Guenther and John Walter Lee, Applications of topological transversality to differential equations. I. Some nonlinear

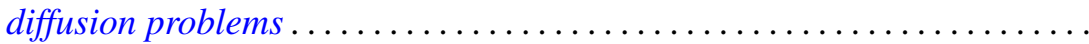

David E. Handelman and G. Renault, Actions of finite groups on self-injective

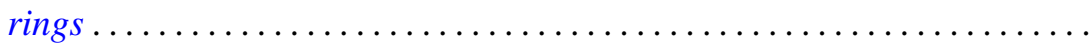

Michael Frank Hutchinson, Local $\Lambda$ sets for profinite groups .............

Arnold Samuel Kas, On the handlebody decomposition associated to a

Lefschetz fibration...

Hans Keller, On the lattice of all closed subspaces of a Hermitian space.....

P. S. Kenderov, Dense strong continuity of pointwise continuous

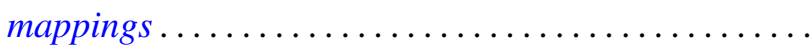

Robert Edward Kennedy, Krull rings.................

Jean Ann Larson, Richard Joseph Laver and George Frank McNulty,

Square-free and cube-free colorings of the ordinals ...

Viktor Losert and Harald Rindler, Cyclic vectors for $L^{p}(G)$

John Rowlay Martin and Edward D. Tymchatyn, Fixed point sets of

1-dimensional Peano continua...

Augusto Nobile, On equisingular families of isolated singularities ...

Kenneth Joseph Prevot, Imbedding smooth involutions in trivial bundles ...

Thomas Munro Price, Spanning surfaces for projective planes in four space.

Dave Riffelmacher, Sweedler's two-cocycles and Hochschild cohomology....

Niels Schwartz, Archimedean lattice-ordered fields that are algebraic over

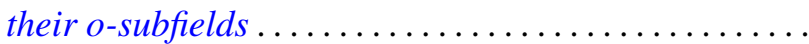

Chao-Liang Shen, A note on the automorphism groups of simple dimension groups.

Kenneth Barry Stolarsky, Mapping properties, growth, and uniqueness of

Vieta (infinite cosine) products ...

Warren James Wong, Maps on simple algebras preserving zero products. I.

The associative case ............................. 